\title{
19 Cholesterol and Alzheimer's disease
}

High cholesterol levels also promote the onset of Alzheimer's disease - the world's most common form of dementia. In Germany, approx. 900,000 of the nearly 1.5 million dementia sufferers have Alzheimer's, which the WHO cites as contributing to $60-70 \%$ of dementia cases worldwide. Besides non-functioning tau proteins in the neurofibrillary bundles, other causes of Alzheimer's come from depositions (plaques) of amyloid-beta, a peptide of 42 amino acids in length ( $\mathbf{A} \boldsymbol{\beta}-\mathbf{4 2})$, which is found mainly in the limbic system, neocortex and hippocampus (Bateman et al. 2012). One function of the hippocampus is converting important information from short-term to long-term memory ( $\triangleright$ Chapter 92 ). The A $\beta-42$ peptide is formed by cleavage of a membrane-bound amyloid precursor protein in the presence of the enzyme gamma-secretase. The gamma-secretase-activating protein increases the activity of gamma-secretase ( $\mathrm{He}$ et al. 2010), but so does cholesterol - with the result that elevated cholesterol levels are often accompanied by increased amyloid plaque formation.
Cleavage of the precursor protein also releases $\mathbf{A} \boldsymbol{\beta}-40$, a peptide two amino acids shorter than $A \beta-42$. This building block plays a positive role in pathogenesis insofar as it throttles cholesterol biosynthesis and thus also indirectly reduces the concentration of neurotoxic $\mathrm{A} \beta-42$ by decreasing gamma-secretase activity. When cholesterol levels are normal, both feedback loops are in equilibrium.

When cholesterol levels are high, however, the protective function gained by lowering $A \beta-40$ cholesterol is often no longer effective enough, and the harmful properties of $\mathrm{A} \beta-42$ predominate. This is even more relevant considering that $A \beta-42$ activates gamma secretase, thereby promoting further cleavage of the pre-amyloid. $A \beta-42$ achieves this indirectly by hindering neurons in the brain from forming sphingomyelin. In fact, sphingomyelin is capable of inhibiting gamma-secretase by itself. However, it can only limit pre-amyloid cleavage when it is present in sufficient concentrations.

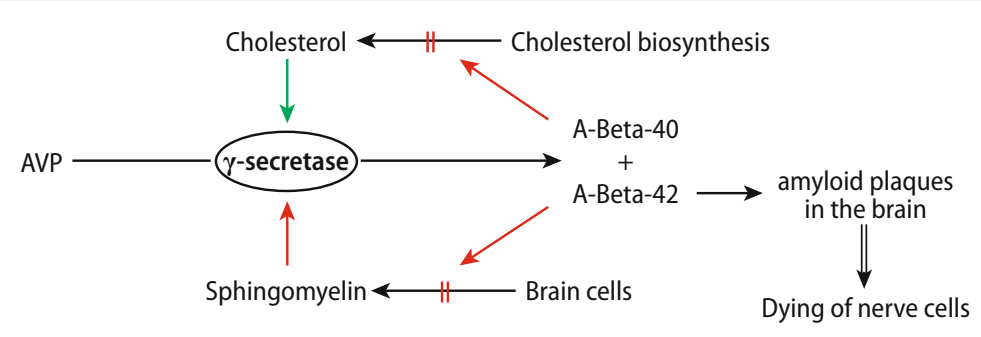

- Fig. 19.1 Amyloid plaques formation: black arrow formation, green arrow activation, red arrow inhibition, APP amyloid precursor protein, A-Beta-40 and -42 APP splice products 